\title{
Motorcycle control-oriented dynamics modeling for accelerated maneuvers on slippy terrains
}

\author{
Elżbieta Jarzębowska ${ }^{1 *}$, Michał Cieśluk ${ }^{1}$ \\ ${ }^{1}$ Warsaw University of Technology, Power and Aeronautical Engineering Dept., 00-665 Warsaw, Nowowiejska 24 st., Poland
}

\begin{abstract}
The paper presents a motorcycle dynamics model developed for testing and future model based controller designs for accelerated maneuvers on variable slip terrains. The dynamics is simplified, yet it captures real motorcycle behaviors when it accelerates and decelerates on a curvy trajectory and contact forces due to variable ground properties change and allow to generate slip. The tire - terrain model is based upon a simplified Pacejka model. The paper objective is not to obtain complex dynamical systems of equations like those required for high-fidelity simulations. Instead, the aim is to derive simple but reliable and manageable models that enable designing and implementing, and verifying control laws, as well as maintaining their capability to capture the main behaviors of real systems. The paper applies the adopted assumptions to develop the motorcycle model, and presents simulation tests for the motorcycle acceleration and deceleration during turn maneuvers, and during changes of the ground the vehicle moves on.
\end{abstract}

\section{Introduction}

Motorcycling is a popular means of transportation in urban areas, used for sports and entertainment, so a number of drivers increase. Many road accidents with motorcyclists involved are reported. The reports show that major accidents are due to human driving errors. Many motorcyclists have little knowledge about the dynamic behavior of their vehicles, about the drivermotorcycle interaction. Driving assistance systems could be the key to better control vehicles. In this context, several active safety systems for motorcycles were developed [1]. Also, the motorcycle is itself an interesting object from dynamics and control point of view.

There are many dynamic models of motorcycles in the literature starting from very simple to very sophisticated and specialized to some modeling goals. A high-fidelity model may accurately reflect the response of a vehicle, but added details may complicate motion planning and control designs. It is then a trade-off between the accuracy of the dynamics model and difficulties of controller designs and their performance. An extensive overview of general modeling concepts and a survey of models used for motion planning and control can be found in $[1,2]$. Also, there are many popular models of mobility for car-like vehicles [3-6]. They are widely used in control and motion planning to approximate a vehicle's behavior in response to control actions. Many of these models are kinematical ones [1]. Many dynamic models are complex, they focus upon the vehicle itself and use either very simple tire - terrain interaction models or assume no slip motion conditions, i.e. introduce nonholonomic constraints into the model. Formally, the nonholonomic constraint limits maneuverability of the vehicle model. A real vehicle due to slip has more degrees of freedom. Since forces that develop between tires and the ground play the significant role in fast motorcycle maneuvers, the main goal of this research is to develop and test a form of simplified control oriented dynamics model with reliable tireground interaction model is welcome.

The paper presents a motorcycle dynamics model developed for testing and future model based controller designs for accelerated maneuvers on variable slip terrains. The dynamics is simplified, yet it captures real motorcycle behaviors when accelerates and decelerates on a curvy trajectory and contact forces due to variable ground properties are allowed to change, and allow to generate slip. The tire - terrain model is based upon a Pacejka model [2] but it is simplified. The adopted simplifications proved to be satisfactory for reproducing the motorcycle behaviors. The motivations for this research are multiple. When designing control strategies and algorithms for wheeled vehicles like mobile robots, cars or motorcycles, the development of mathematical models that describe the dynamics of real systems is of a significant importance. It allows using model-based approaches to controller designs, which achieve better implementation of control laws and their better performance. In these cases, the objective of modeling is not to obtain complex dynamical systems of equations like those required for high-fidelity simulations. Instead, the aim is to derive simple but reliable and manageable models that enable designing and implementing, and verifying control laws, as well as maintaining, at the

Corresponding author: elajarz@meil.pw.edu.pl 
same time, their capability to capture the main behaviors of real systems.

The research contribution is twofold. It is a motorcycle dynamics model developed for testing and future model based controller designs for accelerated maneuvers on variable slip terrains and a simplified tire terrain Pacejka model, which satisfactory reproduce the motorcycle behaviors.

The paper is organized as follows. Section 1 provides some introductory comments about the motorcycle reported research. Section 2 presents the adopted assumptions to the motorcycle model and the dynamics model development. Simulation tests for the motorcycle acceleration and deceleration during turn maneuvers and during the change of the ground the vehicle moves on are detailed in Section 3. The paper closes with conclusions and a list of references.

\section{Motorcycle dynamics model}

The motorcycle dynamics model is developed for a vehicle model presented in Fig. 1. It is simplified, yet captures the main bodies the model consists of as well as the contact force due to the change of the mass center of the motorcyclist- motorcycle system.

The following simulation model inputs were taken into account:

- $m_{k}$ - handle bar mass,

- $m_{r}$ - motorcycle frame mass (includes the drives mass),

- $m_{p}, m_{t}$ - front and rear wheel masses,

- $l_{\text {rosi }}$ - distance between the vehicle axes,

- $l_{\text {siedz }}$ - distance between the driver seat and the rear wheel axis,

- $l_{\text {rama }}$ - distance between the frame mass centre and the rear wheel axis,

- $R_{p}$ - radius of the front wheel,

- $R_{t}$ - radius of the rear wheel,

- $h_{\text {siedz }}$ - height of a driver seat (measured from the ground),

- $h_{\text {rama }}$ - distance between the frame mass centre and the ground,

$-\varepsilon$ - yaw angle of the frame,

- $g_{r}$ - prism width, which models the frame, the motorcyclist and the rear wheel,

- $a$ - distance between the front wheel contact point and the vehicle mass center,

- $b$ - distance between the rear wheel contact point and the vehicle mass center,

- $h_{m}$ - height of the mass center location,

- $I_{p x 0}, I_{m x 0}, I_{p z 0}, I_{m z 0}, I_{m x z 0}, I_{w p}, I_{w t^{-}}$moments and products of inertia of the motorcycle and its wheels.

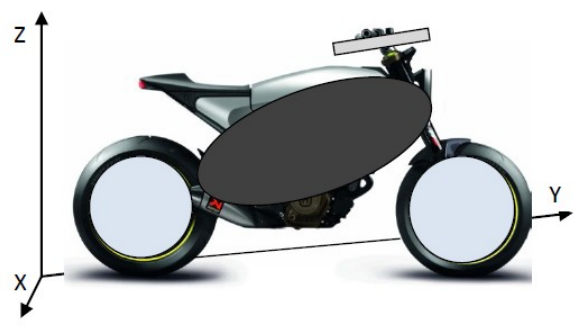

Fig. 1. Motorcycle model.

\subsection{Motorcycle tire-ground interaction forces}

The significant part of the model is the tire - ground interaction model. It is based upon the Pacejka model [2] and its components are presented at a scheme in Fig. 2.

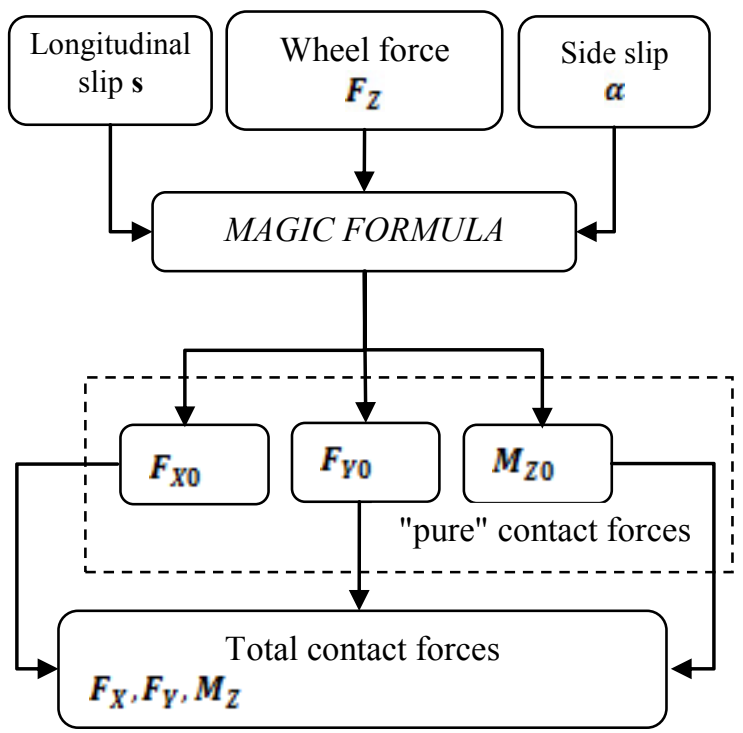

Fig. 2. Tire-ground interaction forces modeling.

The "pure contact forces" mean the forces determined for one slip type. The reference inertial frame $X, Y, Z$ is the right hand side Euclidian.

\subsection{Motorcycle dynamic equations}

The simplified motorcycle dynamics model is developed for the seven degrees of freedom represented by the quasi velocity vector : : lho

The velocities $u, v, r$, are the velocities along the $x, y, z$, body-fixed frame, and the angles $\varphi, \delta, \theta_{P}, \theta_{T}$ are the motorcycle bank angle, handle bar heading angle, yaw angles of the front and rear wheels, respectively. The Lagrange equations of motion developed for the motorcycle model have the form

$$
M(\boldsymbol{q}) \cdot \ddot{\boldsymbol{q}}=V_{C O R}(\boldsymbol{q}, \dot{\boldsymbol{q}})+F_{A C T}+F_{P A C}(\boldsymbol{q}, \dot{\boldsymbol{q}})
$$

where the matrices in (1) are as follows:

$$
\boldsymbol{F}_{P A C}=\left\{\begin{array}{c}
F_{x p}+F_{x t}-F_{y p} \cdot \delta \cdot \cos \varepsilon \\
F_{y p}+F_{y t}+F_{x p} \cdot \delta \cdot \cos \varepsilon \\
-b F_{y t}+a F_{y p}+M_{z t}+M_{z p}+\left(t_{c}+a \cos \varepsilon\right) \delta F_{x p} \\
-b F_{y t}+a F_{y p}+M_{x t}+M_{x p}+t_{c} \delta F_{z p} \\
-F_{P X} \cdot r_{T} \\
-F_{L X} \cdot r_{T} \\
-F_{F X} \cdot r_{F}
\end{array}\right\} ;
$$

is the force vector of interaction between the tire and the ground and is determined according to Pacejka model,

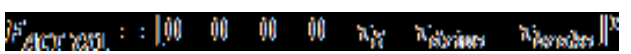

is the external force vector due to a driver, 


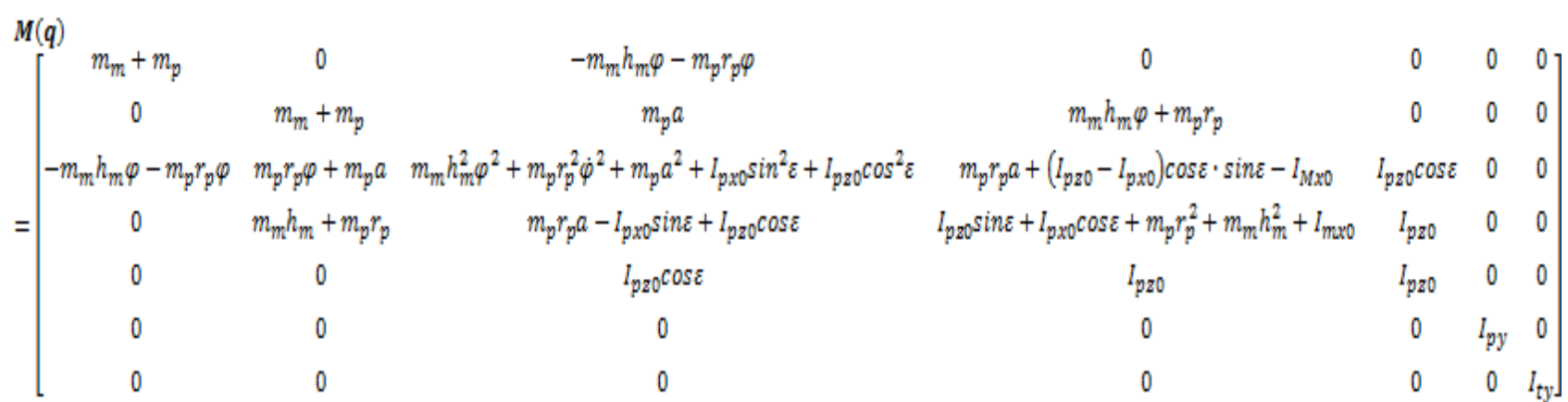

$$
\boldsymbol{V}_{\text {COR }}(\boldsymbol{q}, \dot{\boldsymbol{q}})=\left\{\begin{array}{c}
\delta \cdot \cos \varepsilon+\left(m_{m}+m_{p}\right) r v+m_{p} a r^{2}+2\left(m_{m} h_{m}+m_{p} r_{p}\right) \cdot r \dot{\varphi} \\
-\left(m_{m}+m_{p}\right) u r+\left(m_{m} h_{m}+m_{p} r_{p}\right) \varphi r^{2} \\
-2 r \varphi \dot{\varphi}\left(r_{p}^{2}+h_{m}^{2}\right)-\left(m_{m} h_{m}+m_{p} r_{p}\right) \varphi v r-a m_{p} u r+\varphi g\left(m_{m} h_{m}+m_{p} r_{p}\right) \\
0 \\
0 \\
0 \\
0
\end{array}\right\}
$$

In Eq. (1) adhesion forces are projected onto the vehicle principle axes. Forces that act upon the wheels are adhesive force and moments of forces regulated by a motorcyclist. The moments are due to the handle bar rotation, propulsion from the rear wheel and the braking torque of the front wheel. The dynamics model (1) encounters for the motorcycle stabilizing effect due to the so called dragging effect for the front wheel. It is measured by the distance between the contact point of the front wheel and the point of intersection of the ground plane with the handlebar rotation axis.

\section{Simulation studies - accelerated motorcycle maneuvers on slippy roads}

Three driving scenarios are tested in simulation studies. They are selected as "everyday driving maneuvers". They demonstrate both the effectiveness of the developed motorcycle dynamics model and effects of accelerated motion when variable terrain - tire interactions are accounted for.

\subsection{Scenario 1 - constant velocity motion along a circle}

Motorcycle moves along a circle with a constant velocity and a constant handle bar angle.

In all scenarios the following data is used (all are SI units): $m_{r}+m_{k}=278, m_{p}=10, \mathrm{~m}_{\mathrm{t}}=11, I_{p x 0}=0.44, I_{p z 0}=0.44$, $I_{m x 0}=170.02, I_{m z 0}=5.55, I_{m x z 0}=2.59, I_{w p}=0.88, I_{w t}=1.16$.

The trajectories of the front and rear wheels, and of the mass center are shown in Fig. 3.

Figure 4 presents aligning torques (top) and the camber torques for the front and rear wheels.

Figure 5 presents longitudinal slips to the front and rear wheels (top) and the side slip angle to these wheels for the scenario 1 conditions.

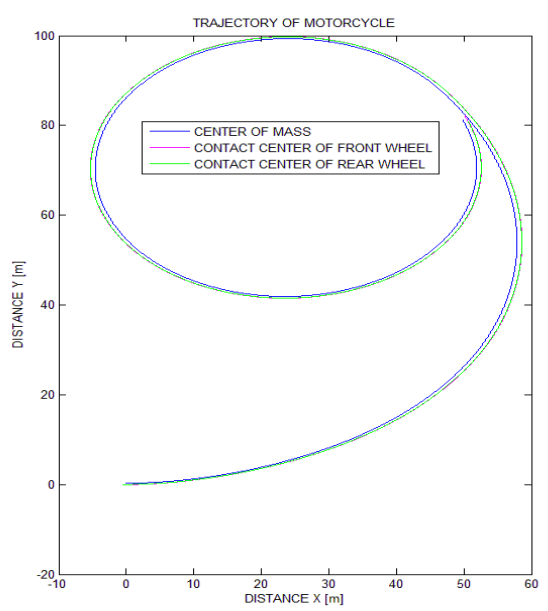

Fig. 3. Trajectories of the front, rear wheels and the mass center in scenario 1 .
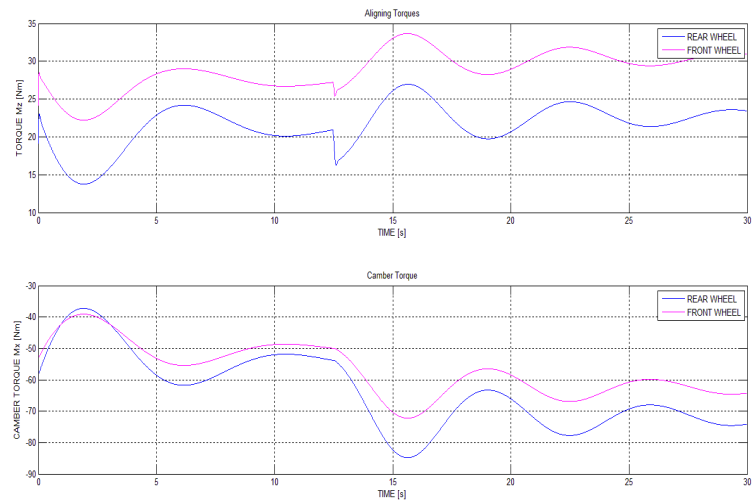

Fig. 4. Aligning and camber torques for a motorcycle. 

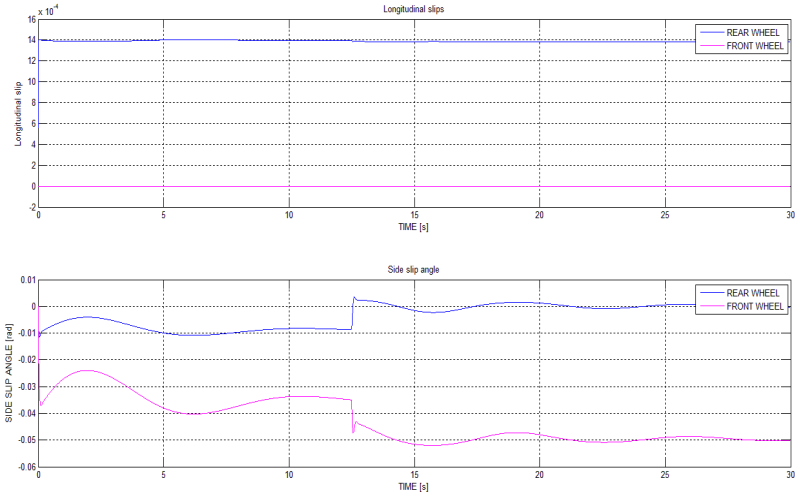

Fig. 5. Longitudinal slips to the front and rear wheels (top) and the side slip angle to the wheels in scenario 1 .

\subsection{Scenario 2 - accelerated turning and the change of a surface friction}

In this case, the motorcycle turns and accelerates when turning. Suddenly, a driver notices an obstacle and starts decelerating after $1 \mathrm{~s}$ (the reaction time for a motorcyclist is assumed to be $1 \mathrm{~s}$ ). But the surface changes in this $1 \mathrm{~s}$ (friction coefficient decreases about 0.5). This scenario is for example for a case of oil spot, which was not seen by a motorcyclist.

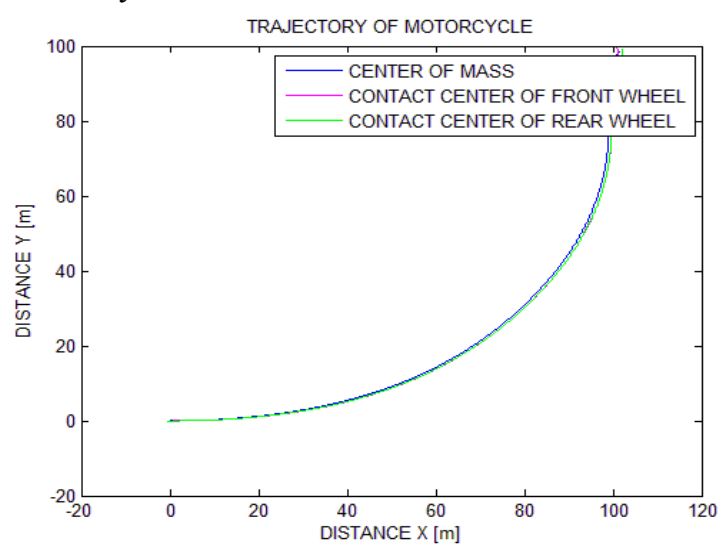

Fig. 6. Trajectories of the front, rear wheels and the mass center in scenario 2 .

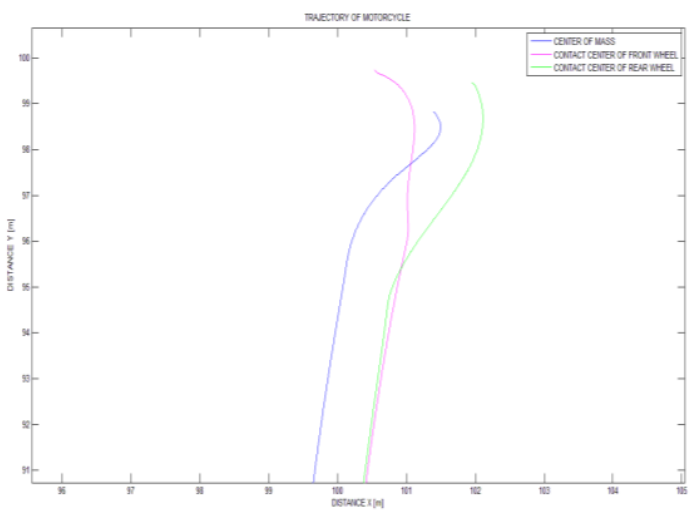

Fig. 7. Trajectories of the front, rear wheels and the mass center in scenario 2 - magnified.
The motorcycle falls; see fig. 6 and 7. Notice that the adhesive side forces almost vanishes during braking, and it contributes to falling.

Figures 8 to 11 present side and longitudinal forces as well as side slip angle and longitudinal slips, vertical loads on the front and rear wheels, and side and longitudinal velocities associated to the maneuver.

Each motorcycle due to its design and performance properties has a specified maneuver related longitudinal velocity, handle bar heading angle and its bank angle. Simulation studies presented in these three scenarios start from setting these variables at some initial magnitudes. Between 2 to 7 seconds of motion, the motorcycle accelerates what manifests in the growing longitudinal force on the rear wheel. At the same time, side forces on both wheels grow; see Fig. 8. Between 7 to 9 seconds, the motorcycle does not accelerate. Braking starts at the second 9. Next, according to our scenario, after 1 second the friction coefficient drops from 1.2 to 0.7 . It causes significant delay of the braking wheel. It results in increase of longitudinal slip magnitude; see Fig.9. The adhesion force is directed along the wheel axis and the lack of the side component of this force results in the motorcycle falling; see Fig. 11.
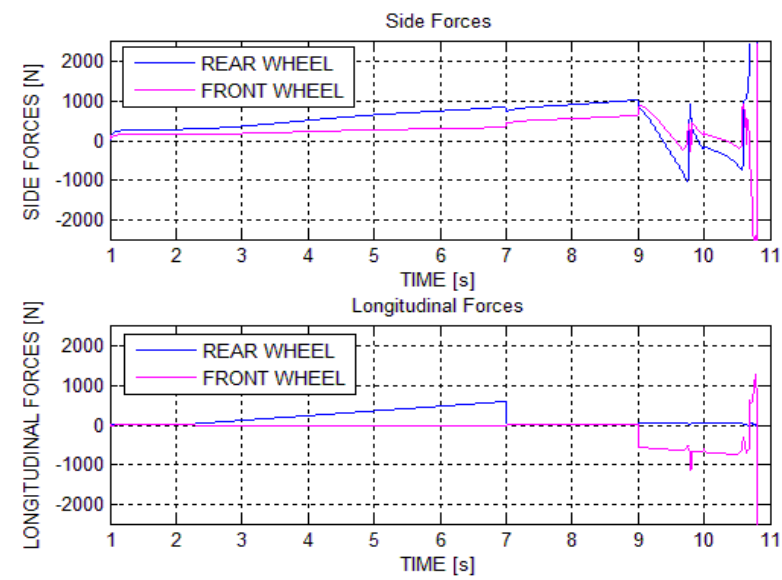

Fig. 8. Side and longitudinal forces on the wheels in scenario 2.
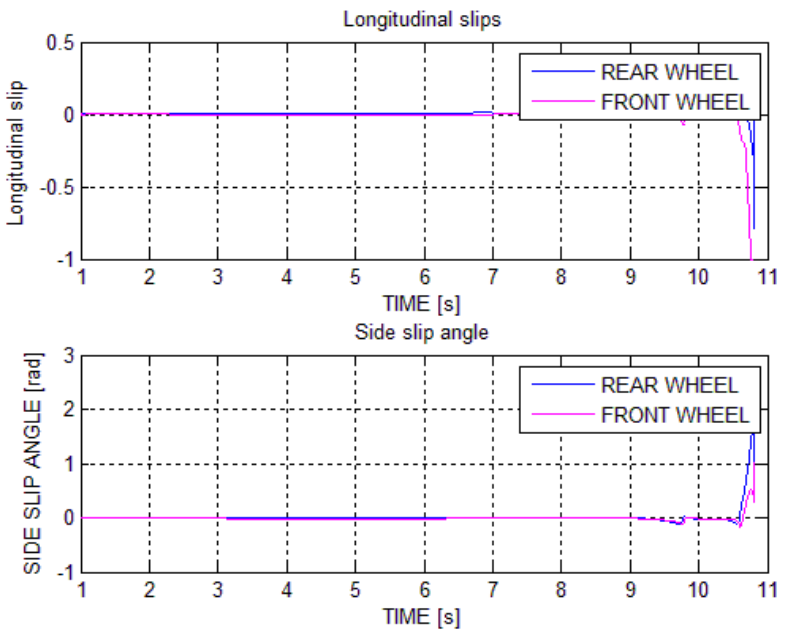

Fig. 9. Longitudinal slip to the front and rear wheels (top) and the side slip angle to the wheels in scenario 2 . 


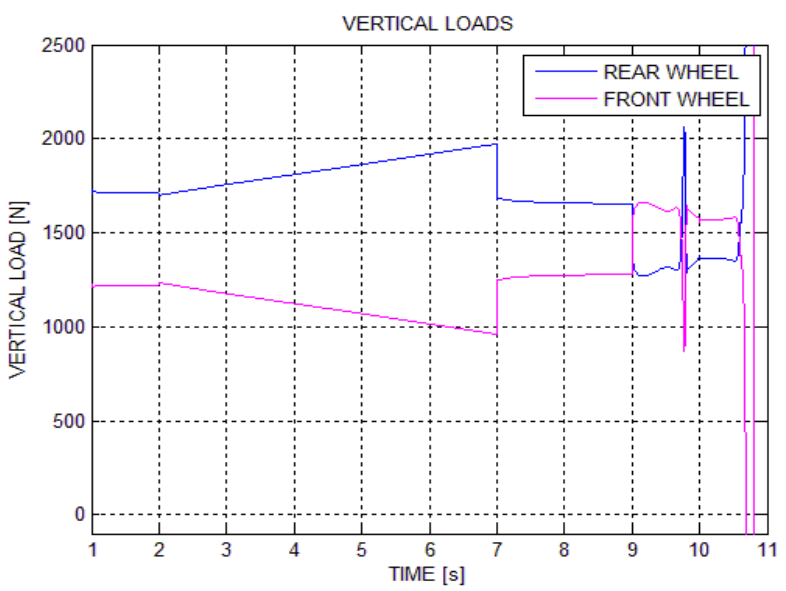

Fig. 10. Vertical loads on the motorcycle wheels in scenario 2.
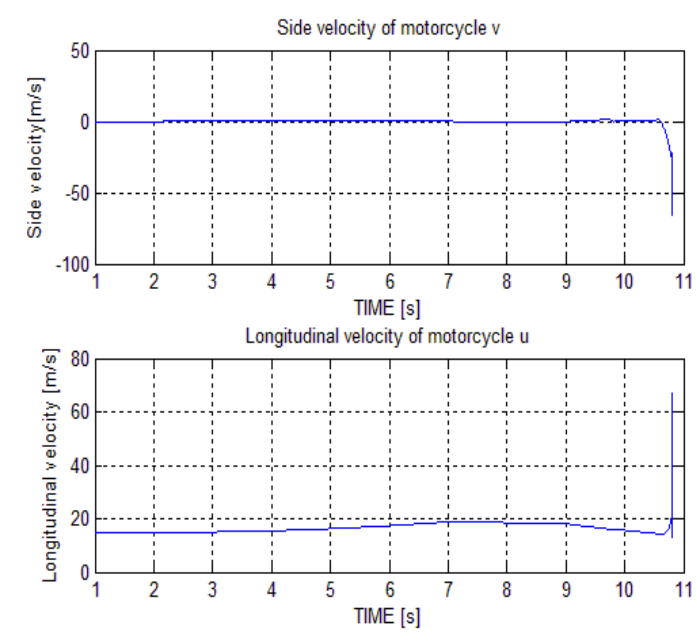

Fig. 11. Side and longitudinal velocities of the motorcycle.

\subsection{Scenario 3 - accelerated turning and no change of a surface friction}

The scenario 3 looks like scenario 2 but the surface the motorcycle drives on does not change. The motorcyclist can survive this time and does not fall. Figures 12 and 13 present the trajectories of the wheels and the vehicle mass center.

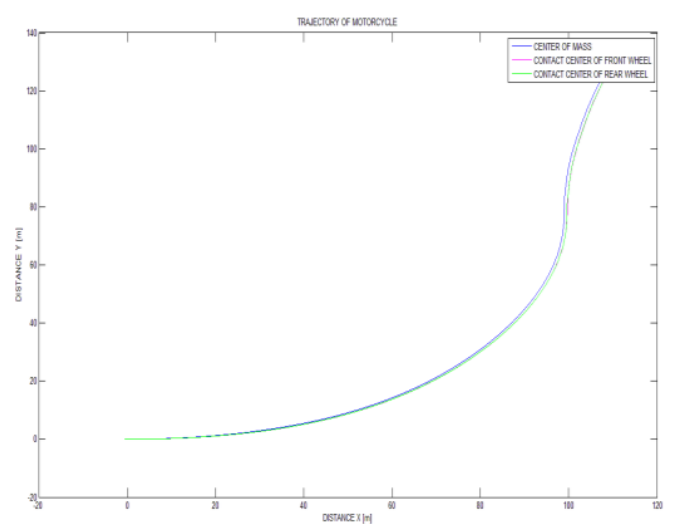

Fig. 12. Trajectories of the front, rear wheels and the mass center in scenario 3 .

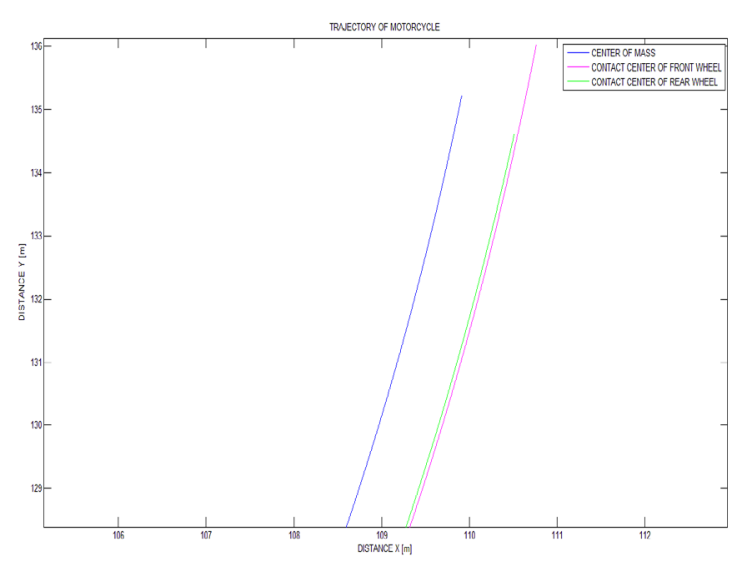

Fig. 13. Trajectories of the front, rear wheels and the mass center in scenario 3 - magninfied.

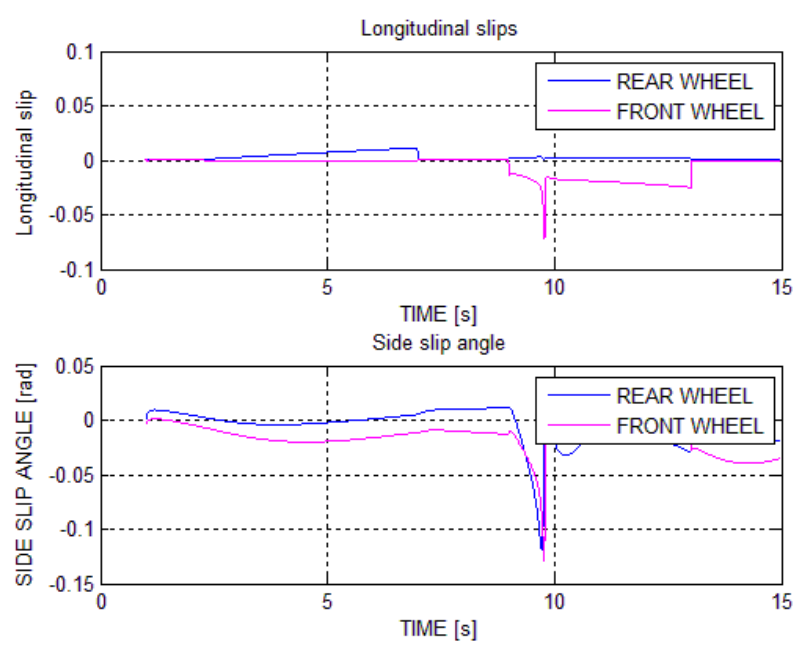

Fig. 14. Longitudinal slips to the front and rear wheels (top) and the side slip angle to the wheels in scenario 3.

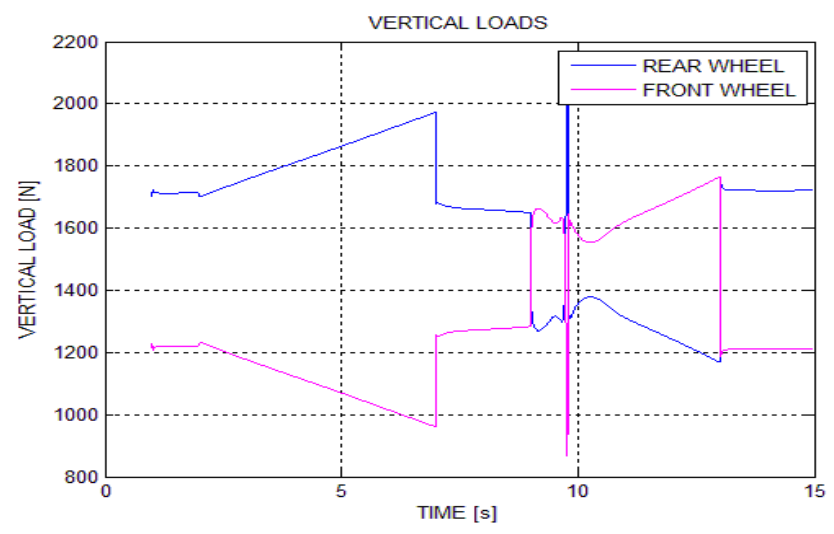

Fig. 15. Vertical loands on the wheels in the driving scenario 3.

Comparing scenarios 2 and 3 , the motorcycle accelerates and then brakes in the region, at which the longitudinal slip does not increase. Also, adhesion forces are uniformly distributed. Longitudinal acceleration magnitude, force exerted by the wheels onto the ground and adhesion forces are all related. Braking causes the force increase on the front wheel and accelerating on the the rear wheel. The vertical load on the motorcycle 
wheels is always symmetric with respect to half of its total weight; see Fig. 15.

\section{Conclusions}

The paper presents a motorcycle simplified dynamics model oriented to test accelerated maneuvers on variable slip terrains and for future model based controller designs for such maneuvers. The dynamics is simplified, yet it captures real motorcycle behaviors when it accelerates and decelerates on slippy terrains. Three simulation scenarios are presented. They reproduce real situations motorcyclists may experience. The simulation tests for the motorcycle model acceleration and deceleration during turn maneuvers, and during the change of the ground the vehicle moves on confirmed good agreement of the model outcomes with real motorcycle behaviors. Future research is planned to design a controller for motorcycle driving and training, and test it experimentally.

\section{References}

1. B. Paden, M. Cáp, S. Z. Yong, D. Yershov, and E. Frazzoli, A Survey of Motion Planning and Control Techniques for Self-driving Urban Vehicles, IEEE Trans. Intel. Vehicles, 1(1), pp. 33-55 (2016).

2. H. Pacejka, 2012, Tire and Vehicle Dynamics (SAE Intern. and Butterworth Heinemann, 2012)

3. G. Franke, W. Suhr, and F Rieß, An Advanced Model of Bicycle Dynamics, European J. Physics, 11 (2), pp. 116-121 (2000).

4. U. Kumar and N. Sukavanam, Dynamic Modeling and Tracking Control of a Four Wheeled Nonholonomic Mobile Robot, Proc. 13th Int. Conf. Mechanics and Machines, (NaCoMM07), pp. 127134 (2007).

5. D. Limebeer and R.S. Sharp, Bicycles, Motorcycles and Models, IEEE Control Syst., 26 (5), pp. 34-61 (2006).

6. JP. Meijaard, J.M. Papadopoulos, A. Ruina, and A.L. Schwab, Linearized Dynamics Equations for the Balance and Steer of a Bicycle: A Benchmark and Review, Proc. Royal Soc. A: Math., Phys. Eng. Sci., 463 (2084), pp. 1955-1982 (2007). 